\title{
Small-signal Stability Assessment of Heterogeneous Multi-converter Power Systems
}

\author{
Yuhan Zhou, Huanhai Xin, Di Wu, Feng Liu, Zhiyi Li, Guanzhong Wang, Hui Yuan, Ping Ju
}

\begin{abstract}
The increasing penetration of renewable resources into the power network through grid-following converters has increased the risks of oscillation instability. In such a power system, it is challenging for assessing the small-signal stability due to the complex interaction among converters interconnected through the power network. Moreover, the assessment complexity is further increased in a heterogeneous multi-converter system, where the interconnected converters have different control configurations or parameters from different manufacturers. To tackle the challenges, this paper proposes a method for the small-signal stability analysis of a heterogeneous multi-converter system. To this end, it is first theoretically proved that the small-signal stability of a heterogeneous system can be characterized by an equivalent homogeneous one, where all interconnected converters have the same control configurations and parameters. This can reduce the complexity of the small-signal stability assessment of the original heterogeneous system by decoupling the equivalent homogeneous system into a set of subsystems. To further reduce the assessment complexity, it is derived that the small-signal stability and stability margin of the heterogeneous system can be estimated based on the smallest eigenvalue of a weighted Laplacian matrix of the power network. Based on the analysis results, a scalable method is developed for assessing the smallsignal stability of heterogeneous multi-converter systems. The efficacy of the proposed method is validated by performing both modal analysis and time-domain simulations on two heterogeneous multiple-converter systems with different network sizes and converter numbers.
\end{abstract}

Index Terms-Small-signal stability, heterogeneous system, homogeneous system, grid-following converters.

\section{INTRODUCTION}

The increasing integration of renewable energy resources such as wind and solar into the electric power grid through power electronic converters is changing grid dynamics and challenging grid planning and operation. Particularly, the growing penetration of grid-following converters via phaselock loops (PLL) under weak grid connections has resulted in emerging multi-frequency oscillation instability risks [1]-[7]. Since the oscillation stability issues belong to the small-signal stability category, their mechanism and characteristics have been mainly investigated by the following techniques: timedomain analysis method [8]-[9], modal analysis method [10][11], and impedance-based analysis method [12]-[15]. The essence of the time-domain analysis method in [8]-[9] is to solve the nonlinear electromagnetic transient simulation model and judge the stability of the system based on the oscillation

Y. Zhou, H. Xin, Z. Li, G. Wang, H. Yuan, P. Ju are with the college of electrical engineering, Zhejiang university, Hangzhou 310027, China (Email: xinhh@zju.edu.cn); trajectory of the time-domain waveform. When several hundreds of converters are connected to the power grid, the numerical dimensions will significantly surge, and the computational efficiency will sharply decrease, which may greatly limit the applicability of the time-domain analysis method. The modal analysis method in [10]-[11] is based on the eigen-solution of state matrix of the system linearized statespace model. In a multi-converter system where there are $n$ converters and each converter has $m$ state variables, when the converter number $n$ is too large, the dimensions of the system state matrix (i.e., $\mathrm{nm}$ ) may be too high to be numerically handled for modal analysis. The impedance-based analysis method in [12]-[15] considers negative effective resistance of multiple converters as the potential oscillation sources in the system. However, a large number of possible oscillatory sources in the system to be screened may make impedancemodel-based analysis inapplicable, and the obtained stability results may be too conservative in the practical system. Thus, it is challenging to use the above three methods for small-signal stability analysis in a power system with a large number of converters.

To address the challenges, different solutions have been proposed. In [16]-[18], the capacity-weighted mean value method was proposed to create the aggregated converters, but this method does not consider the dynamic equivalence between the aggregated converter model and the original multiconverter system. As a result, the resulting aggregated model may cause incorrect results for the small-signal stability assessment. In [18]-[20], the parameter identification method was developed to minimize the dynamic difference between the multi-converter system and the aggregated converter system, but this method is often computationally complicated due to the requirement for detailed information about the dynamics of a multiple-converter system. Instead of relying on the aggregated converter model, a feasible method was proposed in [21] for the impedance-based analysis by decoupling a power system with a large number of converters into a set of equivalent singleconverter subsystems. This decoupling method allows that the small-signal stability analysis experience for a single-converter system can easily be transplanted to a multi-converter system. Following the idea, a reduced-order method for modal analysis was proposed in [10] for a multi-converter power system. However, these decoupling methods in [10] and [21] mainly focus on a homogeneous system, where all converters have similar control configurations and parameters. A practical

D. Wu is with the department of electrical and computer engineering, North Dakota State University, Fargo, 58102, USA.

F. Liu is with the State Key Laboratory of Power System and the Department of Electrical Engineering, Tsinghua University, Beijing, 100084, China. 
power system is usually a heterogeneous system, since the integrated converters may have different control configurations or parameters from different manufacturers. In such a heterogeneous system, the interaction among the interconnected converters and the power network become more complex than those in a homogeneous system. This increases the complexity of the small-signal stability assessment, and the above existing decoupling methods are not applicable to such a heterogeneous system. To address this issue, a heuristic method was proposed in [15] to approximate the small-signal dynamics of a heterogeneous multi-converter system by many homogeneous systems so that each homogeneous system can still be decomposed into a set of single-converter systems for the small-signal stability assessment. But this method is derived based on empirical reasoning and abundant tests instead of theoretical justification. As a result, the proposed stability analysis criterion is a sufficient condition rather than a sufficient and necessary one, which may result in relatively conservative stability assessment results on a practical power system with a large number of grid-following converters.

In this paper, we present a method for the small-signal stability assessment of a heterogeneous system with multiple grid-following converters based on modal decoupling theory and matrix perturbation theory. The major contributions of this paper can be summarized as follows:

1) By means of modal decoupling theory and matrix perturbation theory, it is theoretically proved that a heterogeneous multi-converter system can be equivalently converted into a homogeneous multi-converter system and then decouple it into a set of equivalent single-converter subsystems to reduce the complexity of small-signal stability analysis of the original heterogeneous system.

2) On this foundation, it is derived that the small-signal stability and stability margin of a heterogeneous multiconverter system can be estimated based on the smallest eigenvalue of a weighted Laplacian matrix of the power network in the heterogeneous multi-converter system. This further reduces the complexity of the small-signal stability assessment of the heterogeneous system.

3) Based on the analysis results, a scalable method is developed for assessing the small-signal stability of heterogeneous multi-converter systems. It is verified that the proposed method is independent of network size and topologies as well as converter numbers in heterogeneous multi-converter systems by modal analysis and timedomain simulations on two heterogeneous multi-converter systems.

The rest of this paper is organized as follows. Section II presents the modeling of a heterogeneous multi-converter system. On this basis, the small-signal stability of the heterogeneous system is analyzed in Section III. In Section IV, the proposed method is presented. In Section V, the efficacy of the proposed method is demonstrated by modal analysis and time-domain simulations on two test systems with different network topologies and converter numbers. In Section VI, the conclusions are drawn.

\section{II.MODELING OF HETEROGENEOUS MULTI-CONVERTER SYSTEMS}

Let us consider a heterogeneous system with multiple gridfollowing converters (hereinafter are referred to as the converters) shown as Fig. 1, in which there are $n$ converter nodes, $m$ interior nodes (non-converter nodes in the power network), and an infinite node connected to the external grid.

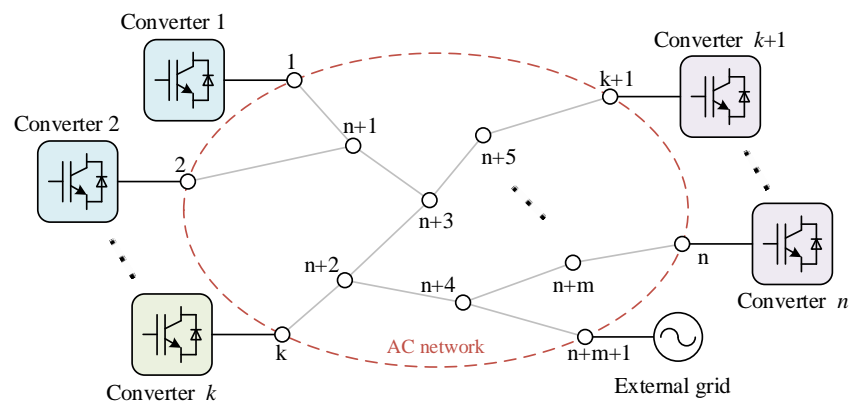

Fig. 1 A power system with $n$ converters.

\section{1) Converter modeling}

The state-space model of the $i^{\text {th }}$ converter can be represented

$$
\left\{\begin{array}{l}
\frac{d}{d t} \Delta \boldsymbol{X}_{i}=\boldsymbol{A}_{i} \Delta \boldsymbol{X}_{i}+\boldsymbol{B}_{i} \Delta \boldsymbol{U}_{i} \\
\Delta \boldsymbol{I}_{i}=S_{B i} \cdot \boldsymbol{C}_{i} \Delta \boldsymbol{X}_{i}
\end{array}\right.
$$

where $\Delta$ denotes the small increment of a variable vector; $\boldsymbol{X}_{i} \in$ $\mathbf{R}^{p \times 1}$ is the vector of all the state variables of $i^{\text {th }}$ converter; $p$ denotes the number of the state variables; $\boldsymbol{I}_{x y y}=\left[\begin{array}{ll}I_{x, i} & I_{y, i}\end{array}\right]^{T}$ and $\boldsymbol{U}_{x y i}=\left[U_{x, i} U_{y, i}\right]^{T}$ are the output current and terminal voltage at the point of interconnection of $i^{\text {th }}$ converter, respectively, expressed in the common $x-y$ coordinate; $\boldsymbol{A}_{i} \in \mathbf{R}^{p \times p}$ and $\boldsymbol{B}_{i} \in$ $\mathbf{R}^{p \times 2}$ are the state matrix and control matrix of $i^{\text {th }}$ converter, respectively; $\boldsymbol{C}_{i} \in \mathbf{R}^{2 \times p}$ denotes the output matrix of $i^{\text {th }}$ converter, which is normalized by $S_{B i}$, and $S_{B i}$ represents the ratio of $i^{\text {th }}$ converter's rated capacity to the capacity base value of the system when performing per-unit calculations. Hence, the statespace model of $i^{\text {th }}$ converter can be denoted briefly as $\left(\boldsymbol{A}_{i}, \boldsymbol{B}_{i}, \boldsymbol{C}_{i}\right.$, $\left.S_{B i}\right)$. Since the following discussion is not related to the specific form of $\boldsymbol{A}_{i}, \boldsymbol{B}_{i}$, and $\boldsymbol{C}_{i}$, their detailed derivations are not provided in this section due to the limitation of space.

Based on (1), the transfer function matrix of the system with $n$ converters can be formulated as:

$$
\left[\begin{array}{c}
\mathbf{0}^{P \times 1} \\
\Delta \boldsymbol{I}_{x y}
\end{array}\right]=\left[\begin{array}{cc}
\operatorname{diag}\left(s \mathbf{I}-\boldsymbol{A}_{i}\right) & \operatorname{diag}\left(-\boldsymbol{B}_{i}\right) \\
\operatorname{diag}\left(S_{B i} \cdot \boldsymbol{C}_{i}\right) & \mathbf{0}^{2 n \times 2 n}
\end{array}\right]\left[\begin{array}{c}
\Delta \boldsymbol{X} \\
\Delta \boldsymbol{U}_{x y}
\end{array}\right], i=1, \ldots, n
$$

where symbol $\operatorname{diag}(\cdot)$ represents a diagonal matrix; $s$ represents the Laplace operator; $\boldsymbol{I}$ denotes an identity matrix with a proper dimension; and $\mathbf{0}$ represents null matrices; $\Delta \boldsymbol{X}=\left[\Delta \boldsymbol{X}_{1}{ }^{T} \ldots \Delta \boldsymbol{X}_{n}{ }^{T}\right]^{T}$; $\Delta \boldsymbol{I}_{x y}=\left[\Delta \boldsymbol{J}_{x y 1}{ }^{T} \ldots \Delta \boldsymbol{I}_{x y n}{ }^{T}\right]^{T} ; \quad \Delta \boldsymbol{U}_{x y}=\left[\Delta \boldsymbol{U}_{x y 1}{ }^{T} \ldots \Delta \boldsymbol{U}_{x y n}{ }^{T}\right]^{T} ; \quad$ and $P$ represents the total number of all the converters' state variables.

By using the Kron reduction [23], $\Delta \boldsymbol{X}$ in (2) can be eliminated and thus the dynamics of all converters can be represented as

$$
\Delta \boldsymbol{I}_{x y}=-\operatorname{diag}\left[S_{B i} \cdot \boldsymbol{C}_{i}\left(s \mathbf{I}-\boldsymbol{A}_{i}\right)^{-1} \boldsymbol{B}_{i}\right] \Delta \boldsymbol{U}_{x y}, i=1, \ldots, n
$$




\section{2) Power network modeling}

For the line connecting nodes $i$ and $j$ in the power network, its dynamic equation can be expressed as:

$$
\begin{aligned}
& {\left[\begin{array}{l}
\Delta I_{x, i j} \\
\Delta I_{y, i j}
\end{array}\right]=\frac{1}{\omega_{0} L_{i j}} \gamma(s)\left[\begin{array}{c}
\Delta U_{x i}-\Delta U_{x j} \\
\Delta U_{y i}-\Delta U_{y j}
\end{array}\right],} \\
& \gamma(s)=\frac{1}{(s+\tau)^{2} / \omega_{0}+\omega_{0}}\left[\begin{array}{cc}
s+\tau & \omega_{0} \\
-\omega_{0} & s+\tau
\end{array}\right]
\end{aligned}
$$

where $\omega_{0}$ is the rated angular velocity of the ac grid; $L_{i j}$ is the inductance between nodes $i$ and $j$; $\tau$ is the ratio of the line resistance to line inductance (i.e., $\tau=R / L$ ); $\left[\Delta I_{x, i j}, \Delta I_{y, i j}\right]^{T}$ is the current vector flowing from nodes $i$ to $j$ in the common $x-y$ coordinate.

When all lines in the power network have the identical ratio $\tau$, the network dynamics of the multi-converter power system can be formulated as:

$$
\Delta \boldsymbol{I}_{x y}=\left[\boldsymbol{Q}_{\text {red }} \otimes \gamma(s)\right] \Delta \boldsymbol{U}_{x y}
$$

where symbol $\otimes$ denotes the Kronecker product; $\boldsymbol{Q}_{\text {red }}$ is the reduced-order grounded Laplacian matrix only with $n$ converter nodes, and its specific derivation is provided in Appendix A.

\section{3) Modeling of heterogeneous multi-converter power system}

Based on the converter modeling in (3) and the power network modeling in (5), the closed-loop characteristic equation of the heterogeneous multi-converter system can be represented:

$$
\begin{aligned}
0 & =\operatorname{det}\left[\boldsymbol{Y}_{\text {het_sys }}(s)\right] \\
& =\operatorname{det}\left[\operatorname{diag}\left[\boldsymbol{C}_{i}\left(s \mathbf{I}-\boldsymbol{A}_{i}\right)^{-1} \boldsymbol{B}_{i} \cdot \boldsymbol{\gamma}(s)^{-1}\right]+\boldsymbol{Y}_{e q} \otimes \mathbf{I}_{2}\right]=0
\end{aligned}
$$

where $\boldsymbol{Y}_{\text {het__sys }}(s)$ represents the closed-loop transfer function of the heterogeneous system; symbol $\operatorname{det}[\cdot]$ denotes the determinant function; $\boldsymbol{Y}_{e q}=\boldsymbol{S}_{B}{ }^{-1} \boldsymbol{Q}_{\text {red }}$ represents the weighted Laplacian matrix; $\boldsymbol{S}_{\mathrm{B}}=\operatorname{diag}\left(S_{B i}\right), i=1, \ldots, n ; \mathbf{I}_{2}$ denotes a 2 -by-2 identity matrix. The system is small-signal stable if and only if all the solutions of (6) (i.e., system eigenvalues) are located in the left-half of the complex plane.

\section{SMall-Signal Stability ANALYSIS OF HETEROGENEOUS MULTI-CONVERTER POWER SYSTEMS}

In this section, the previous results of the small-signal stability analysis of homogeneous multi-converter systems will first be briefly introduced. The analysis results will be then extended to heterogeneous multi-converter systems for the small-signal stability analysis based on modal decoupling theory and matrix perturbation theory.

\section{A. Small-signal Stability Analysis of Homogeneous Systems}

For a homogeneous system with $n$ converters, each converter has the same dynamic control strategy and parameters. That is, $\boldsymbol{A}_{i}=\boldsymbol{A}, \boldsymbol{B}_{i}=\boldsymbol{B}, \boldsymbol{C}_{i}=\boldsymbol{C}(i=1, \cdots, n)$. Under this condition, (6) can be rewritten as (7) below for the small-signal stability.

$$
\begin{aligned}
0 & =\operatorname{det}\left[\boldsymbol{Y}_{\text {hom_sys }}(s)\right] \\
& =\operatorname{det}\left[\mathbf{I}_{n} \otimes\left(\boldsymbol{Y}_{C B R} \cdot \boldsymbol{\gamma}(s)^{-1}\right)+\boldsymbol{Y}_{e q} \otimes \mathbf{I}_{2}\right]
\end{aligned}
$$

$$
\boldsymbol{Y}_{C B R}(s)=\boldsymbol{C}\left(s \mathbf{I}-\boldsymbol{A}_{i}\right)^{-1} \boldsymbol{B}
$$

where $\boldsymbol{Y}_{\text {hom_sys }}(s)$ represents the closed-loop transfer function of the homogeneous multi-converter system; $\boldsymbol{Y}_{C B R}(s)$ denotes the open-loop transfer function of each converter port in a homogeneous system; $\mathbf{I}_{n}$ denotes a $n$-by- $n$ identity matrix.

According to [21], it is known that matrix $\boldsymbol{Y}_{e q}$ is diagonalizable and its all eigenvalues are positive. Thus, there is a matrix $\boldsymbol{W}$ that can decompose matrix $\boldsymbol{Y}_{e q}$ into a diagonal matrix in which the diagonal elements consist of the eigenvalues $\left(\sigma_{i}, i=1, \ldots, n\right)$ in the order of $0 \leqslant \sigma_{1} \leqslant \sigma_{2} \cdots \leqslant \sigma_{n}$. That is,

$$
\boldsymbol{W}^{-1} \boldsymbol{Y}_{e q} \boldsymbol{W}=\boldsymbol{\Lambda}=\operatorname{diag}\left(\sigma_{i}\right) \quad i=1, \ldots, n
$$

Combining (9) with (7) yields,

$$
\begin{aligned}
& 0=\operatorname{det}\left[\left(\boldsymbol{W}^{-1} \otimes \mathbf{I}_{2}\right) \boldsymbol{Y}_{\text {hom } \_s y s}(s)\left(\boldsymbol{W} \otimes \mathbf{I}_{2}\right)\right] \\
& \Leftrightarrow 0=\prod_{i=1}^{n} \operatorname{det}\left[\boldsymbol{Y}_{C B R}(s) \cdot \gamma(s)^{-1}+\sigma_{i} \cdot \mathbf{I}_{2}\right]
\end{aligned}
$$

Equation (10) shows the complexity of the small-signal stability analysis of the homogeneous multi-converter system can be reduced by two features:

First, the dynamics of the homogeneous system with $n$ converters can be characterized by $n$ dynamically independent subsystems, and each subsystem has a converter connected to the infinite node via a line with the equivalent susceptance equal to eigenvalue $\sigma_{i}$. Thus, the homogeneous system is stable if and only if all the equivalent subsystems are stable.

Second, the small-signal stability of the homogeneous multiconverter system can be characterized by the smallest eigenvalue of the weighted Laplacian matrix $\boldsymbol{Y}_{e q}$ of the power network. Since each converter has the same control configuration and parameters in the homogeneous system, it is know from (10) that the stability of each subsystem depends on $\sigma_{i}(i=1, \ldots, n)$ when the control configuration and parameters are given. The smaller $\sigma_{i}$ means its corresponding subsystem is more likely to be unstable. In other words, the small-signal stability of the entire homogeneous system depends on the most critical subsystem which has the smallest eigenvalue $\sigma_{1}$ of matrix $\boldsymbol{Y}_{e q}$ as the equivalent line susceptance connected to the grid. Since the stability of the most critical subsystem depends on the smallest eigenvalue $\sigma_{1}$, the small-signal stability of the entire homogeneous system depends on the smallest eigenvalue $\sigma_{1}$, which is defined as the generalized short-circuit ratio for grid strength assessment in [21].

\section{B. Small-signal Stability Analysis of Heterogeneous Systems}

For a heterogeneous system with $n$ converters, each converter may have different control configurations or parameters. That is, $\boldsymbol{A}_{m, i} \neq \boldsymbol{A}_{m, j}, \boldsymbol{B}_{m, i} \neq \boldsymbol{B}_{m, j}$, and $\boldsymbol{C}_{m, i} \neq \boldsymbol{C}_{m, j}, i \neq j$, and $i, j=1, \ldots, n$. Under this condition, the characteristic equation (6) cannot be decoupled, so those two natures of homogeneous systems cannot be extended to the heterogeneous system for reducing the complexity of the small-signal stability analysis. To address this issue, we first construct an equivalent homogeneous system, and then prove that the equivalent homogeneous system can characterize the small-signal stability of the original heterogeneous system. 
For the equivalent homogeneous system, its characteristic equation can be represented by

$$
\begin{gathered}
\operatorname{det}\left[\overline{\boldsymbol{Y}}_{\text {hom_sss }}(s)\right]=\operatorname{det}\left[\mathbf{I}_{n} \otimes\left(\overline{\boldsymbol{Y}}_{C B R}(s) \gamma(s)^{-1}\right)+\boldsymbol{Y}_{e q} \otimes \mathbf{I}_{2}\right]=0 \\
\overline{\boldsymbol{Y}}_{C B R}(s)=\sum_{i=1}^{n} p_{i} \cdot \boldsymbol{C}_{i}\left(s \mathbf{I}-\boldsymbol{A}_{i}\right)^{-1} \boldsymbol{B}_{i}
\end{gathered}
$$

where $\overline{\boldsymbol{Y}}_{\text {hom_sss }}(s)$ represents the closed-loop transfer function of the equivalent homogeneous system; $\overline{\boldsymbol{Y}}_{C B R}(s)$ denotes the openloop transfer function of each converter port in the equivalent homogeneous system; $p_{i}=u_{i 1} v_{i 1}$ is the participation factor that quantifies the participation degree of the $i^{\text {th }}$ converter to the smallest eigenvalue $\sigma_{1}$ of matrix $\boldsymbol{Y}_{e q}$ and thus characterize the impact of the $i^{\text {th }}$ converter on the small-signal stability of the system ${ }^{[21]} ; u_{i 1}$ and $v_{i 1}$ are the $i^{\text {th }}$ element of normalized left and right eigenvectors $\boldsymbol{u}_{1}{ }^{T}$ and $\boldsymbol{v}_{1}$ for the smallest eigenvalue $\sigma_{1}$ of matrix $\boldsymbol{Y}_{e q}$, respectively.

The Proposition III.1 below proves that the small-signal stability of the original heterogeneous system can be characterized by the equivalent homogeneous system.

Proposition III.1 Define $\lambda_{j}(s)$ and $\bar{\lambda}_{j}(s) \quad(j=1,2)$ are the dominant eigenvalue function of the heterogeneous system and its equivalent homogeneous system, respectively, and they are pertinent to the two systems' dominant eigenvalues. Then, the loci of $\lambda(s)$ and $\bar{\lambda}(s)$ have the following relationship:

$$
\lambda_{j}(s)=\bar{\lambda}_{j}(s)+\boldsymbol{O}\left(\left\|\boldsymbol{Y}_{\text {het_sys }}(s)-\overline{\boldsymbol{Y}}_{\text {hom } \_s y s}(s)\right\|\right) j=1,2
$$

where $\boldsymbol{O}(\cdot)$ denotes the second-order and much higher order approximate error of a function; $\|$.$\| denotes the norm of any$ matrix.

Proof: Based on the small-signal stability analysis of the homogeneous system in Section III.A, it is known that $\bar{\lambda}_{j}(s)$ can be obtained from the most critical subsystem decoupled from the homogeneous system. Thus, $\bar{\lambda}_{j}(s)$ can be formulated:

$$
\begin{aligned}
\bar{\lambda}_{j}(s) & =\left(\boldsymbol{u}_{1} \otimes \boldsymbol{\psi}_{j}^{T}(s)\right) \overline{\boldsymbol{Y}}_{\mathrm{hom}_{-} s y s}(s)\left(\boldsymbol{v}_{1} \otimes \boldsymbol{\tau}_{j}(s)\right) \\
& =\alpha_{j}(s)+\sigma_{1} \quad(j=1,2)
\end{aligned}
$$

where $\boldsymbol{\psi}(s)=\left[\begin{array}{ll}\boldsymbol{\psi}_{1}(s) & \boldsymbol{\psi}_{2}(s)\end{array}\right]$ and $\tau(s)=\left[\begin{array}{ll}\boldsymbol{\tau}_{1}(s) & \boldsymbol{\tau}_{2}(s)\end{array}\right] ; \boldsymbol{\psi}_{j}^{T}(s)$ and $\tau_{j}(s)(j=1,2)$ represent the normalized left and right eigenvectors of $\overline{\boldsymbol{Y}}_{C B R}(s) \gamma(s)^{-1} \quad$ corresponding to the characteristic function $\alpha_{j}(s)(j=1,2)$, and they satisfy $\boldsymbol{\psi}_{j}^{T}(s) \tau_{j}(s)=1,(j=1,2)$.

In a heterogeneous multi-converter system, $\boldsymbol{Y}_{\text {het_sys }}(s)$ can be considered as a result of imposing a perturbation on
$\overline{\boldsymbol{Y}}_{\text {hom_sys }}(s)$. According to matrix perturbation theory (Theorem 2.3 in [24]), the dominant eigenvalue function $\lambda_{j}(s) \quad(j=1,2)$ for $\boldsymbol{Y}_{\text {het } \_s y s}(s)$ can be represented as (15) at the bottom of this page.

This completes the proof $\boldsymbol{m}$.

Based on Proposition III.1, the two features of a homogeneous multi-converter system as discussed in Section III. A can be used to reduce the complexity of the small-signal stability of a heterogeneous multi-converter system through its equivalent homogeneous system. Particularly, according to the analysis in Section III. A, it is known that the equivalent homogeneous multi-converter can be decoupled into a set of subsystems for the small-signal stability analysis, and the smallsignal stability of the homogeneous multi-converter system can be characterized by the smallest eigenvalue $\sigma_{1}$ of the weighted Laplacian matrix of the power network. Since the stability of the heterogeneous system can be characterized by its equivalent homogeneous system, the smallest eigenvalue $\sigma_{1}$ can also be used to characterize the small-signal stability of the heterogeneous system. More specifically, the smallest eigenvalue $\sigma_{1}$ is related to the most critical subsystem decoupled from the equivalent homogeneous system, and the characteristic equation of the most critical subsystem can be represented:

$$
\operatorname{det}\left[\overline{\boldsymbol{Y}}_{C B R}(s) \gamma(s)^{-1}+\sigma_{1} \cdot \mathbf{I}_{2}\right]=\bar{\lambda}_{1}(s) \cdot \bar{\lambda}_{2}(s)=0
$$

Based on (16), the stability boundary of the heterogeneous system can be evaluated. Let us define $\sigma_{\mathrm{c}}$ as the critical value of $\sigma_{1}$ when the most critical subsystem reaches the critically stable state. Then, the value of $\sigma_{\mathrm{c}}$ can be obtained by

$$
\sigma_{c}=\underset{\sigma_{1}}{\arg }\left\{\operatorname{det}\left[\overline{\mathbf{Y}}_{C B R}\left(s_{c}\right) \cdot \boldsymbol{\gamma}\left(s_{c}\right)^{-1}+\sigma_{1} \cdot \mathbf{I}_{2}\right]=0\right\}
$$

where symbol $\arg \{\}$ is used to calculate the roots of the equation; $s_{c}=j \omega_{c}$ is the dominant eigenvalues of the system, which are just on the imaginary axis.

The small-signal stability and stability margin of a heterogeneous system can be identified by comparing the $\sigma_{1}$ with $\sigma_{\mathrm{c}}$. That is, the heterogeneous system is stable if and only if the smallest eigenvalue $\sigma_{1}$ is larger or equal to its critical value $\sigma_{\mathrm{c}}$ (i.e., $\sigma_{1} \geqslant \sigma_{\mathrm{c}}$ ). Moreover, the stability margin of the heterogeneous system can be estimated by the difference between $\sigma_{1}$ and $\sigma_{\mathrm{c}}$ (i.e., $\sigma_{1}-\sigma_{\mathrm{c}}$ ).

$$
\begin{aligned}
\lambda_{j}(s) & =\left(\boldsymbol{u}_{1} \otimes \boldsymbol{\psi}_{j}^{T}(s)\right) \boldsymbol{Y}_{\text {het_sys }}(s)\left(\boldsymbol{v}_{1} \otimes \boldsymbol{\tau}_{j}(s)\right)+\boldsymbol{O}\left(\left\|\boldsymbol{Y}_{\text {het_ssys }}(s)-\overline{\boldsymbol{Y}}_{\text {hom_sys }}(s)\right\|\right) \\
& =\boldsymbol{\psi}_{j}^{T}(s)\left[\sum_{i=1}^{n} u_{i 1} v_{i 1} \cdot \boldsymbol{C}_{i}\left(s \mathbf{I}-\boldsymbol{A}_{i}\right)^{-1} \boldsymbol{B}_{i} \cdot \boldsymbol{\gamma}(s)^{-1}\right] \boldsymbol{\tau}_{j}(s)+\sigma_{1}+\boldsymbol{O}\left(\left\|\boldsymbol{Y}_{\text {het_sys }}(s)-\overline{\boldsymbol{Y}}_{\text {hom_sys }}(s)\right\|\right) \\
& =\boldsymbol{\psi}_{j}^{T}(s)\left[\overline{\boldsymbol{Y}}_{C B R}(s) \cdot \boldsymbol{\gamma}(s)^{-1}\right] \boldsymbol{\tau}_{j}(s)+\sigma_{1}+\boldsymbol{O}\left(\left\|\boldsymbol{Y}_{\text {het_sys }}(s)-\overline{\boldsymbol{Y}}_{\text {hom_sys }}(s)\right\|\right) \\
& =\bar{\lambda}_{j}(s)+\boldsymbol{O}\left(\left\|\boldsymbol{Y}_{\text {het_sys }}(s)-\overline{\boldsymbol{Y}}_{\text {hom_sys }}(s)\right\|\right)(j=1,2)
\end{aligned}
$$




\section{Proposed Method For SMall-Signal Stability} ASSESSMENT OF HETEROGENEOUS MUlTi-CONVERTER POWER SYSTEMS

Based on the analytical results in the previous section, a method is proposed for assessing the small-signal stability and stability margin of heterogeneous multi-converter systems. In a heterogeneous multi-converter system, when the detailed dynamic information of each converter is known, the smallsignal stability and stability margin of the system can be assessed by calculating the smallest eigenvalue $\sigma_{1}$ of matrix $\boldsymbol{Y}_{\text {eq }}$, and the value of $\sigma_{\mathrm{c}}$ based on (17) for their comparison. In a practical power system with high penetration of converters, the dynamic information of each converter may not be easily obtained $^{[15]}$. In such a case, all converters can first be divided into several groups based on the manufacturers or parameters of the converters to ensure the dynamics of converters in each group are similar. Then, a representative converter in each group is selected to obtain its dynamic characteristics by field tests or laboratory tests. Thus, the small-signal stability and stability margin of the practical power system can be evaluated by the method illustrated in Fig. 2. The major steps of the proposed method are summarized:

1) Calculate the smallest eigenvalue $\sigma_{1}$ of matrix $\boldsymbol{Y}_{e q}$, and acquire the left and right eigenvectors $\left(\boldsymbol{u}_{1}{ }^{\mathrm{T}}\right.$ and $\boldsymbol{v}_{1}$ respectively) corresponding to the smallest eigenvalue $\sigma_{1}$. Then, calculate the participation factor $p_{i}=u_{i 1} v_{i 1}$ $(i=1, \ldots n)$ for each converter.

2) Divide all converters into $Q$ groups according to the manufacturers or parameters of converters, and then calculate the sum of the participating factors of the converters in each group by $P_{j}=\sum_{i \subset j} u_{i 1} v_{i 1}(j=1, \ldots, Q)$.

3) Select one representative converter of each group for field tests or laboratory tests and then represent the dynamic characteristics of all converters in each group by $\left(\boldsymbol{A}_{j}, \boldsymbol{B}_{j}, \boldsymbol{C}_{j}\right), j=1, \ldots, Q$.

4) Calculate the dynamic model of the converters in the equivalent homogeneous system analytically based on $\overline{\boldsymbol{Y}}_{C B R}(s)=\sum_{j=1}^{Q} P_{j} \cdot \boldsymbol{C}_{j}\left(s \mathbf{I}-\boldsymbol{A}_{j}\right)^{-1} \boldsymbol{B}_{j}$.

5) Calculate the value of $\sigma_{\mathrm{c}}$ based on (17) and assess the small-signal stability and stability margin by comparing $\sigma_{1}$ with $\sigma_{\mathrm{c}}$. If $\sigma_{1} \geq \sigma_{\mathrm{c}}$, then the heterogeneous system is small-signal stable. The stability margin can be assessed by $\sigma_{1}-\sigma_{\mathrm{c}}$.

The proposed method can significantly reduce the complexity of the small-signal stability assessment of heterogeneous multi-converter systems compared with the traditional modal analysis based on state-space model in the time domain. In the traditional modal analysis, the full-order electromagnetic transient model is commonly required for the stability assessment. When each converter in a heterogeneous system with $n$ converters has $m$ state variables, the order of the required model is $n m$. Since the number $n$ of converters is too large in a practical power system, the traditional modal analysis may have a dimension disaster problem. Such a problem can be avoided in the proposed method since the small-signal stability and stability margin of the heterogeneous multi-converter system can be assessed by the smallest network eigenvalue $\sigma_{1}$ and its critical value $\sigma_{\mathrm{c}}$. The critical value $\sigma_{\mathrm{c}}$ can be analytically obtained based on the most critical subsystem rather than the original heterogeneous system. Moreover, the proposed method is scalable since it is independent of network size and topology as well as converter numbers in the heterogeneous system, which will be demonstrated in the next section.

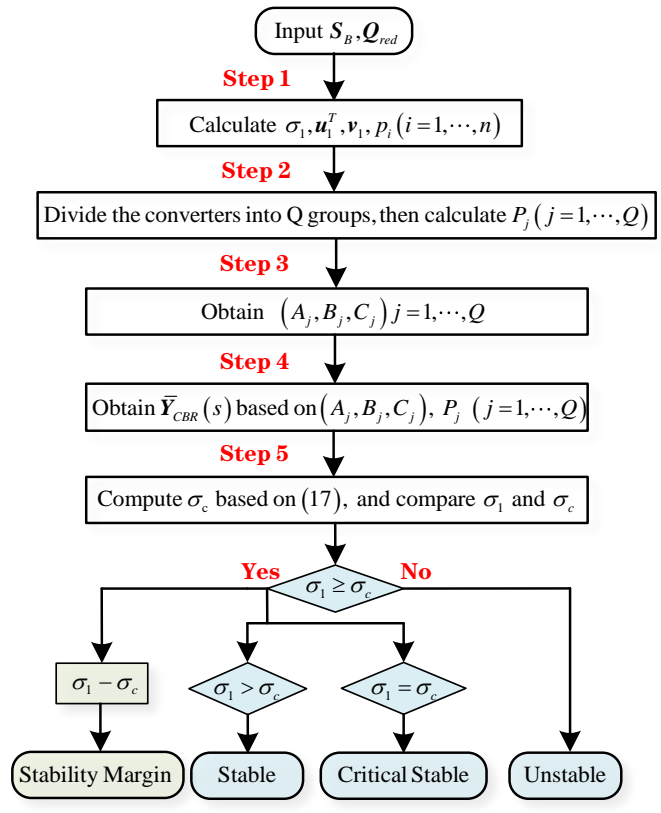

Fig. 2 The process of the practical application process

\section{V.CASE STUDIES}

In this section, the proposed method for the small-signal stability assessment of heterogeneous multi-converter power systems will be validated by the modal analysis and the electromagnetic transient time-domain simulation on a 9converter heterogeneous power system with a meshed network and a 33-converter heterogeneous power system with a radian network.

\section{A. Case studies on a 9-converter heterogeneous power system}

In this heterogeneous system, 9 grid-following converters are interconnected via a 39-bus meshed network as shown in Fig. 3 , which is the same as the one in the standard IEEE 39-bus system, and the line reactance parameters can be found in [25]. In the 9-converters system, converters $1 \sim 6$ use DC voltage outer-loop control mode ( $\mathrm{U}_{\mathrm{dc}}$ control), and converters 7 9 adopt power outer-loop control mode (P control). The control block diagram and parameters of converters are shown in Fig. 4 and Table B.I in Appendix B, respectively. The capacity of each converter in the 9-converter system is 1.0 p.u. Particularly, we set the PLL Proportional-Integrational (PI) control parameters of converters 1 2 as " 29,7500 ", converters $3 \sim 4$ as " 35,7200 ", converters $5 \sim 6$ as " 40,7000 ", and converters $7 \sim 9$ as " 26,7800 ". When the proposed method described in Fig. 2 is used for the small-signal stability assessment in the system, all the converters in the 9-converter heterogeneous system are divided into 4 groups according to their control parameters and control modes. 


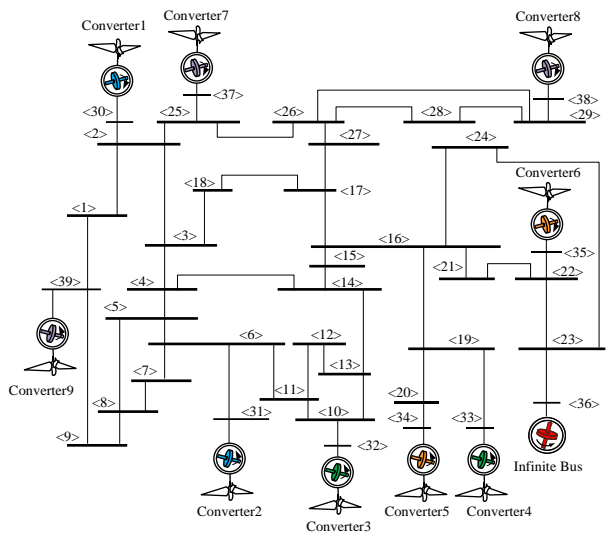

Fig. 3 The 9-converter heterogeneous system with a mesh network topology

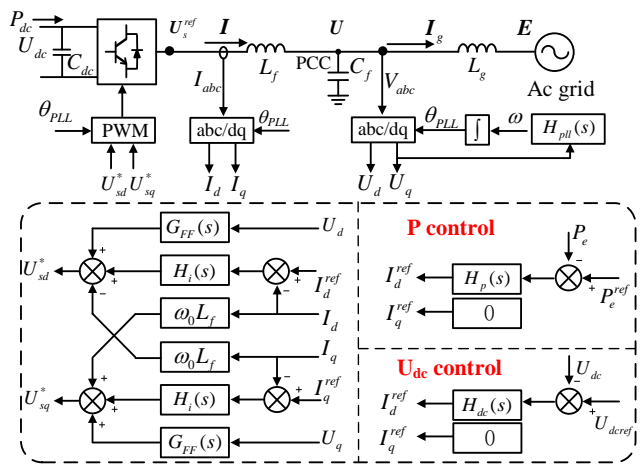

Fig. 4 The control block diagram of converters used in the 9-converter heterogeneous system

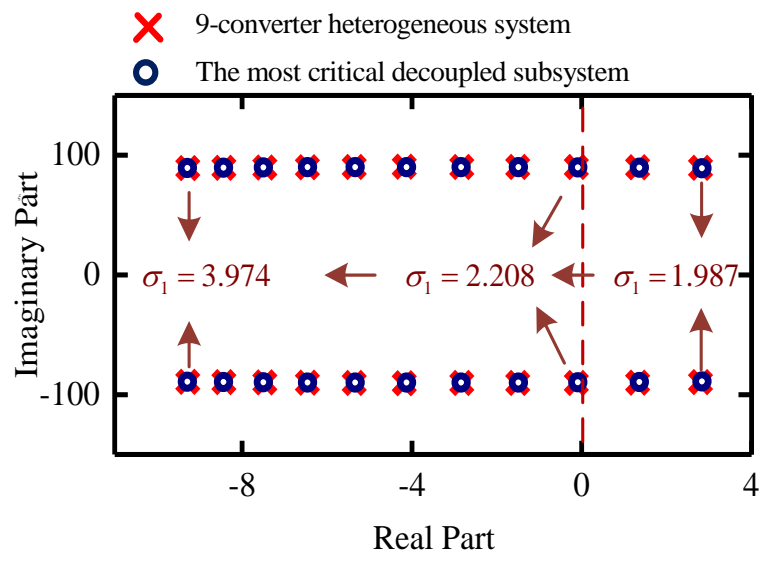

(a)

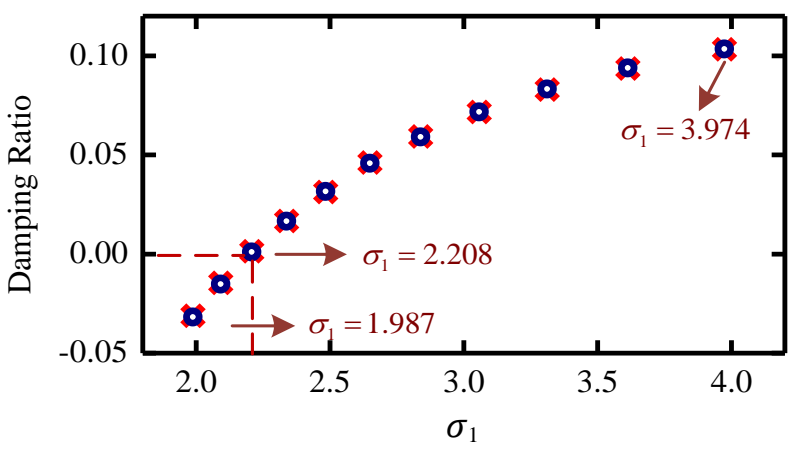

(b)

Fig. 5 The dominant eigenvalues and the damping ratios of the dominant eigenvalues in the 9-converter heterogeneous system and the most critical subsystem when equally proportionally increasing line coefficient $k$ from 0.5 to 1.0 .

\section{1) Modal Analysis}

In the 9-converter heterogeneous system, the proposed method in Fig. 2 is first validated by modal analysis. To this end, several cases are created in the system by equally proportionally changing coefficient $k$, which is proportional to the line length in the per-unit system. When increasing coefficient $k$ from 0.5 to 1.0 , we evaluate the smallest eigenvalues $\sigma_{1}$ of the weighted Laplacian matrix of the power network, the dominant eigenvalues, and the damping ratios of the dominant eigenvalues in the 9-converter heterogeneous system, which are shown in Fig. 5(a) and Fig. 5(b). Also, Fig. 5(a) and Fig. 5(b) show the dominant eigenvalues and the damping ratios of the dominant eigenvalues in the most critical subsystem decoupled from the equivalent homogeneous system of the 9-converter heterogeneous system when increasing coefficient $k$ from 0.5 to 1.0 .

It can be observed from Fig. 5 that the small-signal stability and stability margin of the 9-converter heterogeneous system can be assessed based on the smallest eigenvalues $\sigma_{1}$ and its critical value $\sigma_{\mathrm{c}}$. Based on (17) and the proposed method described in Fig. 2, we can obtain the critical value of $\sigma_{1}$ is 2.208 (i.e., $\sigma_{\mathrm{c}}=2.208$ ). As shown in Fig. 5(a), when $\sigma_{1}=\sigma_{\mathrm{c}}$ (i.e. $\sigma_{1^{-}}$ $\sigma_{\mathrm{c}}=0$ ), the dominant eigenvalues in the 9-converter heterogeneous system are exactly at the imaginary axis, which indicates the system is critically stable. When $\sigma_{1}$ is larger than $\sigma_{\mathrm{c}}\left(\right.$ i.e. $\sigma_{1}-\sigma_{\mathrm{c}}>0$ ), the dominant eigenvalues in the 9-converter heterogeneous system are in the left-half of the complex plane, which suggests the system is stable and has a certain stability margin. When $\sigma_{1}$ is smaller than $\sigma_{\mathrm{c}}$ (i.e. $\sigma_{1}-\sigma_{\mathrm{c}}<0$ ), the dominant eigenvalues in the 9-converter heterogeneous system are in the right-half of the complex plane, which means the system is unstable. The similar observation can also be obtained from Fig. 5(b) in terms of the damping ratio: when $\sigma_{1}=\sigma_{\mathrm{c}}$, the damping ratio of the dominant eigenvalues in the 9-converter heterogeneous system is close to zero, which means that the system is critically stable; when $\sigma_{1}$ is larger (or smaller) than $\sigma_{\mathrm{c}}$, the damping ratio of the dominant eigenvalues in the 9converter system is larger (or smaller) than zero, which indicates the system is stable (or unstable). Thus, the smallsignal stability and stability margin of the 9-converter heterogeneous system can be identified by comparing the $\sigma_{1}$ with $\sigma_{\mathrm{c}}$.

Also, it can be observed from Fig. 5 that the complexity of the small-signal stability analysis of the heterogeneous 9converter system can be reduced by analyzing the stability of the most critical subsystem decoupled from its equivalent homogeneous system. As shown in Fig. 5, the dominant eigenvalues and the damping ratios of the dominant eigenvalues in the 9-converter heterogeneous system are almost identical with those in the most critical. More specifically, Table I compares the difference of the dominant eigenvalues in the 9converter heterogeneous system and the most critical subsystem (i.e., $s_{1}$ and $s_{2}$ ) when the coefficient $k$ is $0.5,0.9$, and 1.0 . According to [10], (18) below is used to evaluate the error between $s_{1}$ and $s_{2}$. It can be seen from this table that the error between $s_{1}$ and $s_{2}$ is smaller than $1 \%$ in either stable or unstable 
system conditions, which shows that the small-signal stability of the 9-converter heterogeneous system can be characterized by the most critical subsystem very well.

$$
\text { Error }=\frac{\left|s_{2}-s_{1}\right|}{\left|s_{1}\right|} \times 100 \%
$$

Table I Comparison of the dominant eigenvalues of the 9-converter heterogeneous system and the most critical subsystem when $k=0.5,0.9$, and 1.0.

\begin{tabular}{ccccc}
\hline$k$ & $\sigma_{1}$ & $s_{1}$ & $s_{2}$ & Error \\
\hline 0.5 & 3.974 & $-9.277 \pm 89.400 i$ & $-9.285 \pm 89.319 i$ & $0.091 \%$ \\
0.9 & 2.208 & $-0.125 \pm 90.118 i$ & $-0.089 \pm 89.770 i$ & $0.388 \%$ \\
1.0 & 1.987 & $2.796 \pm 89.567 i$ & $2.833 \pm 89.117 i$ & $0.504 \%$ \\
\hline
\end{tabular}

\section{2) Time-domain Simulation}

In the 9-converter heterogeneous system, the proposed based method is further verified by the electromagnetic time-domain simulation using MATLAB/Simulink. When coefficient $k$ is set as $0.5,0.9$, and 1.0 , the smallest eigenvalue $\sigma_{1}$ is evaluated by $\sigma_{1}=3.974,2.208$, and 1.987 accordingly. Under these conditions, a disturbance is applied to the infinite bus in the system. The disturbance causes voltage rise by $10 \%$ at $0.10 \mathrm{~s}$ and then is cleared at $0.12 \mathrm{~s}$. Fig. 6 shows the active power trajectories of all the converters in the 9-converter heterogeneous system following the disturbance when $\sigma_{1}=3.974,2.208$, and 1.987, respectively.

It can still be observed from Fig. 6 that the small-signal stability and stability margin of the 9-converter heterogeneous system can be assessed based on the smallest eigenvalues $\sigma_{1}$ and its critical value $\sigma_{\mathrm{c}}$. When $\sigma_{1}=3.974>\sigma_{\mathrm{c}}=2.208$, the convergence oscillation can be seen, which indicates the original system is stable with certain margin; when $\sigma_{1}=1.987<\sigma_{\mathrm{c}}$, the divergent oscillation can be seen, which means the original system is unstable; and when $\sigma_{1}=2.208=\sigma_{\mathrm{c}}$, the undamped oscillation can be seen, which suggests the system is critically stable. The observations in the time-domain simulation are consistent with those obtained from Fig. 5 by modal analysis, which verifies the effectiveness of the proposed method for the small-signal stability assessment in the heterogeneous systems with a meshed network topology.

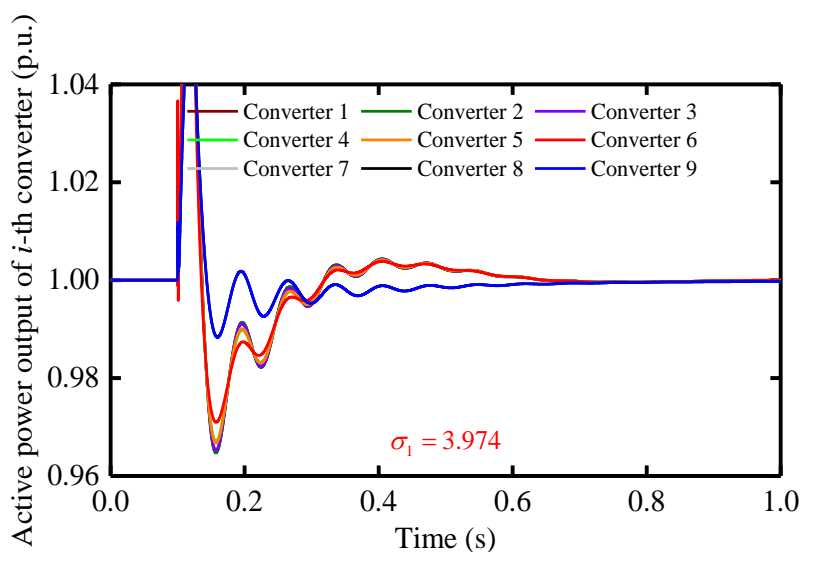

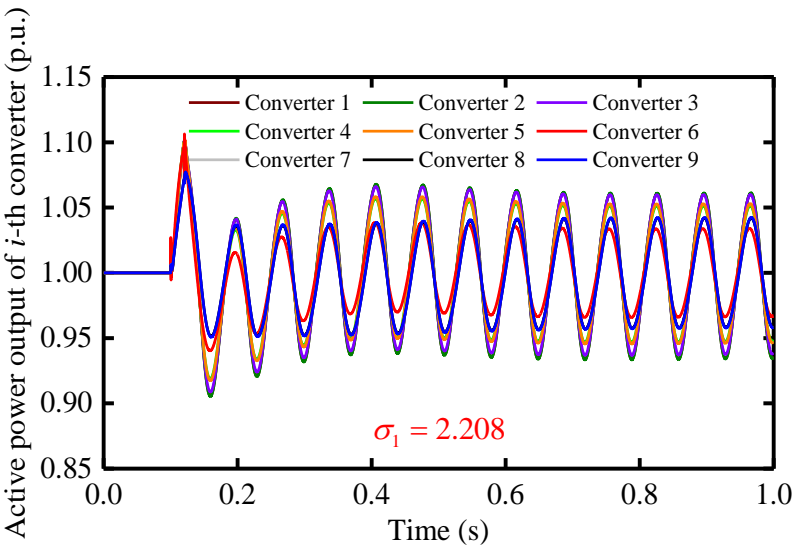

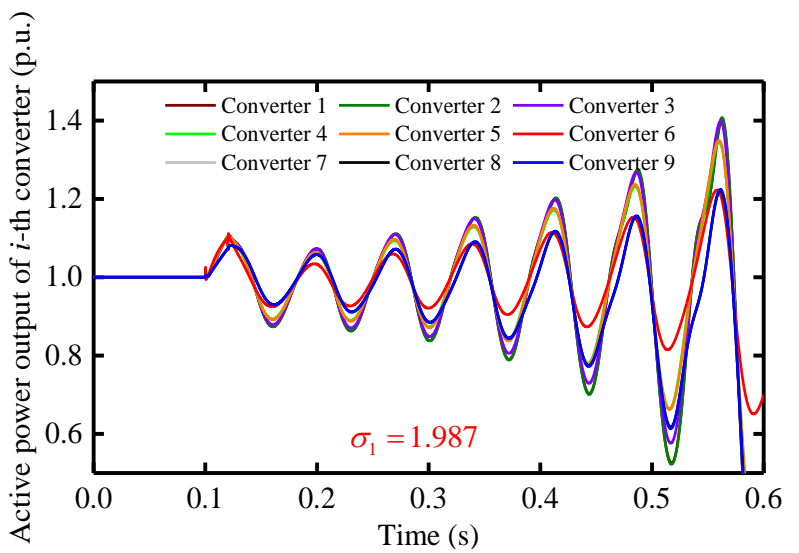

Fig. 6 The active power trajectories of converter 1 in the 9-converter system when $\sigma_{1}=3.974,2.208$, and 1.987 .

\section{B. Case studies on a 33-converter heterogeneous power system}

To demonstrate the proposed method is independent of network size and topology as well as converter numbers in a heterogeneous multi-converter system, the proposed method is also validated on a 33-converter heterogeneous system with a radian network topology, which is shown in Fig. 7. In the system, buses 1 33 are converter nodes connected to the $0.69 \mathrm{kV}$ side of transformers, buses $34 \sim 66$ are connected to the $35 \mathrm{kV}$ side of transformers, and bus 67 is connected to the infinite bus 68 via the $35 \mathrm{kV} / 220 \mathrm{kV}$ transformer. The lines and transformers' parameters in the system can be founded in Table B.II in Appendix B. The control block diagram and parameters of converters are shown in Fig. 4 and Table B.I in Appendix B, respectively. The capacity of each converter in the 33 -converter system is also set as 1.0 p.u. In such a system, converters 1 22 use $\mathrm{U}_{\mathrm{dc}}$ control modes, while converters $23 \sim 33$ use $\mathrm{P}$ control modes. We set the PLL's PI control parameters of converters $1 \sim 11$ in string 1 as " 29,7500 ", converters $12 \sim 22$ in string 2 as " $35,7200 "$, and converters $23 \sim 33$ in string 3 as " 26,7800 ." When the proposed method described in Fig. 2 is used for the small-signal stability assessment in the system, all the converters in the 33-converter heterogeneous system can be divided into 3 groups according to their control parameters and control modes. 


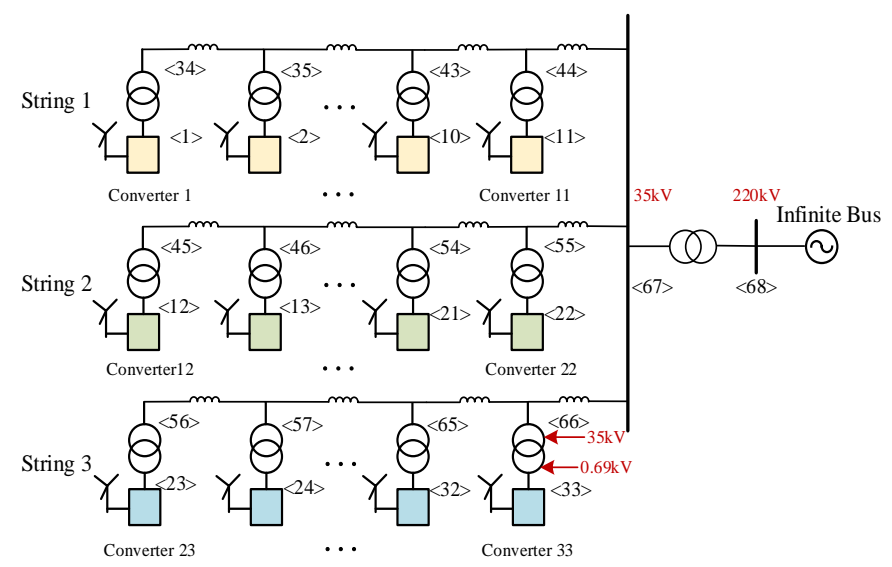

Fig. 7 The 33-converter heterogeneous system with a radian network topology

\section{1) Modal Analysis}

Similar to the verification for the proposed method in the 9converter heterogeneous system, in this 33-converter heterogeneous system, the proposed method is first verified by modal analysis. Several cases are created in the system by only increasing line coefficient $k$ from 2.8 to 5.5. Accordingly, we evaluate the smallest eigenvalues $\sigma_{1}$, the dominant eigenvalues, and the damping ratios of the dominant eigenvalues in the 33converter heterogeneous system. The results are shown in Fig. 8(a) and Fig. 8(b). Also, Fig. 8(a) and Fig. 8(b) show the dominant eigenvalues and the damping ratios of the dominant eigenvalues in the most critical subsystem decoupled from the equivalent homogenous system of the 33-converter heterogeneous system. Table II compares the error of the dominant eigenvalues in the 33-converter heterogeneous system and the most critical subsystem (i.e., $s_{1}$ and $s_{2}$ ) when the coefficient $k$ is $2.8,4.9$, and 5.5. Equation (18) is used to evaluate the error between $s_{1}$ and $s_{2}$.

Similar to the observation from Fig. 5, it can be observed from Fig. 8 that the small-signal stability and stability margin of the 33-converter heterogeneous system can be assessed based on the smallest eigenvalues $\sigma_{1}$ and its critical value $\sigma_{\mathrm{c}}$. Based on (17) and the proposed method described in Fig. 2, we can obtain the critical value of $\sigma_{1}$ is 2.305 (i.e., $\sigma_{\mathrm{c}}=2.305$ ). As shown in Fig. 8, when $\sigma_{1}$ is larger or equal $\sigma_{\mathrm{c}}$, the 33-converter heterogeneous system is stable in terms of its dominant eigenvalues and the damping ratio of the dominant eigenvalues, and the system has a larger stability margin if the positive difference value between $\sigma_{1}$ and $\sigma_{\mathrm{c}}$ (i.e., $\sigma_{1}-\sigma_{\mathrm{c}}$ ) is greater.

Also, it can be observed from Fig. 8 and Table II that the complexity of the small-signal stability analysis of the 33converter system can be reduced by analyzing the stability of the most critical subsystem decoupled from its equivalent homogeneous system, since the 33-converter heterogeneous system and the most critical subsystem have most identical dynamics in terms of their the dominant eigenvalues and the damping ratios of the dominant eigenvalues. Also, Table II shows the errors of the dominant eigenvalues in the 33converter heterogeneous system and the most critical subsystem (i.e., $s_{1}$ and $s_{2}$ ) are smaller than $1 \%$ in either stable or unstable system conditions.

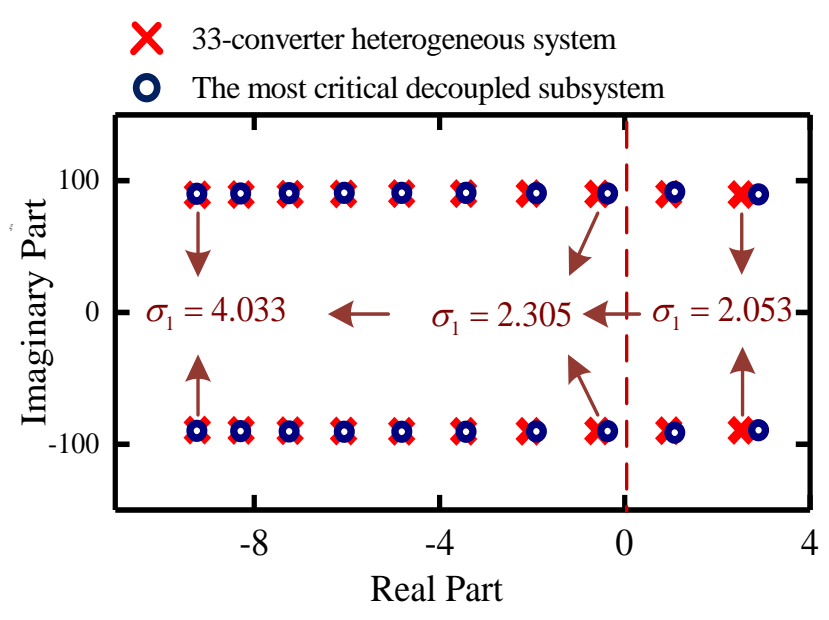

(a)

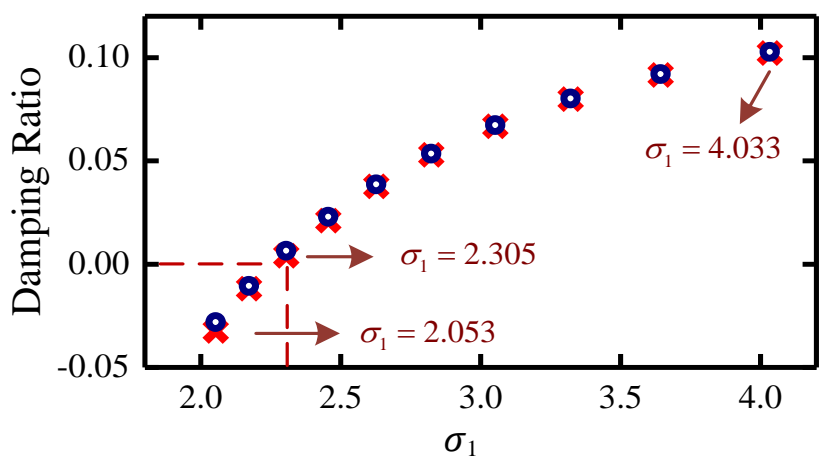

(b)

Fig. 8 The dominant eigenvalues and the damping ratios of dominant eigenvalues in the 33-converter heterogeneous system and the most critical decoupled subsystem when equally proportionally increasing line coefficient $k$ from 2.8 to 5.5

Table II Comparison of the dominant eigenvalues of the 33-converter heterogeneous system and the most critical subsystem when $k=0.57,1.00$, and 1.12 .

\begin{tabular}{ccccc}
\hline$k$ & $\sigma_{1}$ & $s_{1}$ & $s_{2}$ & Error \\
\hline 2.8 & 4.033 & $-9.236 \pm 89.937 i$ & $-9.226 \pm 89.324 i$ & $0.678 \%$ \\
4.9 & 2.305 & $-0.367 \pm 90.342 i$ & $-0.584 \pm 89.998 i$ & $0.450 \%$ \\
5.5 & 2.053 & $2.892 \pm 89.439 i$ & $2.515 \pm 89.417 i$ & $0.422 \%$ \\
\hline
\end{tabular}

\section{2) Time-domain Simulation}

In the 33-converter heterogeneous system, the proposed based method is further verified by the electromagnetic timedomain simulation using MATLAB/Simulink. When coefficient $k$ is set as 2.8, 4.9, and 5.5, the smallest eigenvalue $\sigma_{1}$ is evaluated to obtain $\sigma_{1}=4.033,2.305$, and 2.053 accordingly. Under these conditions, a disturbance is applied to the infinite bus in the system. The disturbance causes voltage rise by $10 \%$ at $0.10 \mathrm{~s}$ and then is cleared at 0.12 s. Fig. 9 shows the output active power trajectories of the overall 33-converter system when $\sigma_{1}=4.033,2.305$, and 2.053, respectively.

Similar to the observation from Fig.6, it can still be observed from Fig.9 that the small-signal stability and stability margin of the 33-converter heterogeneous system can be assessed based on the smallest eigenvalues $\sigma_{1}$ and its critical value $\sigma_{\mathrm{c}}$. When $\sigma_{1}$ is larger (or equal) $\sigma_{\mathrm{c}}$, the 33-converter heterogeneous system is stable since active power trajectories of the overall 33- 
converter heterogeneous system are not divergent. Thus, the system has a certain stability margin when $\sigma_{1}$ is larger than $\sigma_{\mathrm{c}}$. The observations are consistent with those from modal analysis, which validates the proposed method for the small-signal stability assessment in heterogeneous systems with radian network topologies.

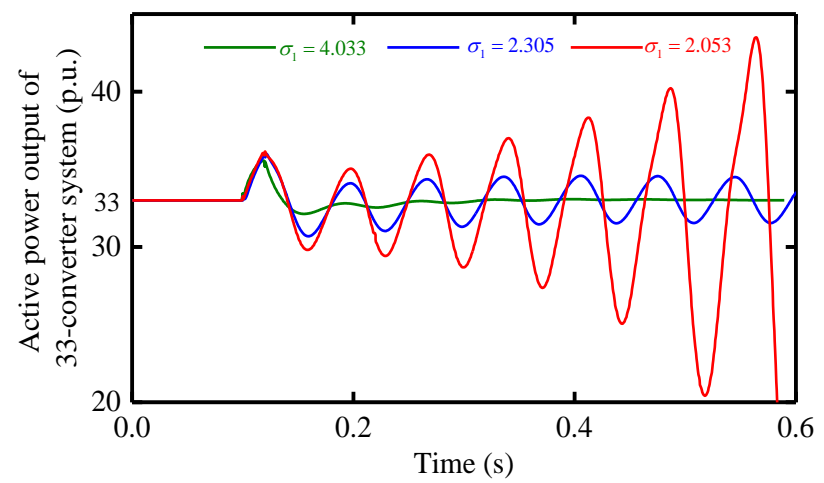

Fig. 9 The active power trajectories of the overall 33-converter heterogeneous system when $\sigma_{1}=4.033,2.305$, and 2.053 .

\section{CONCLUSIONS}

In this paper, a method was proposed for assessing the smallsignal stability of a heterogeneous system with multiple gridfollowing converters. To reduce the assessment complexity of such a system due to the interaction between the power network and the converters with different control configurations or parameters, modal analysis theory and matrix perturbation theory were used to prove that a heterogeneous multi-converter system can be converted into an equivalent homogeneous multi-converter system and then decouple it into a set of subsystems for the small-signal stability assessment. Moreover, the small-signal stability and stability margin of the original heterogeneous system can be estimated based on the smallest eigenvalue of a weighted Laplacian matrix of the power network. Based on the analytical results, a scalable method was proposed and its effectiveness was validated on two heterogeneous multi-converter systems with different network size and topologies as well as converter numbers by modal analysis and time-domain simulations. The proposed method is useful for grid planners and operators to assess the small-signal stability of power systems with large-scale integration of renewable resources.

\section{APPENDIX A}

\section{A. Derivation of $\boldsymbol{Q}_{\text {red }}$ in (5)}

The detailed form of $\boldsymbol{Q}_{\text {red }}$ in (5) can be obtained by eliminating inferior nodes in the multi-converter power system using Kron-reduction shown as follows.

$$
\boldsymbol{Q}_{\text {red }}=\boldsymbol{Q}_{n n}-\boldsymbol{Q}_{n m} \times \boldsymbol{Q}_{m n}^{-1} \times \boldsymbol{Q}_{m m}
$$

where $\boldsymbol{Q}_{n n} \in \mathbf{R}^{n \times n}, \boldsymbol{Q}_{n m} \in \mathbf{R}^{n \times m}, \boldsymbol{Q}_{m n} \in \mathbf{R}^{m \times n}, \boldsymbol{Q}_{m m} \in \mathbf{R}^{m \times m}$ are all submatrices of $\boldsymbol{Q}=\left[\begin{array}{l|l}\boldsymbol{Q}_{n n} & \boldsymbol{Q}_{n m} \\ \hline \boldsymbol{Q}_{m n} & \boldsymbol{Q}_{m m}\end{array}\right] ; \boldsymbol{Q} \in \mathbf{R}^{(n+m) \times(n+m)}$ is the grounded Laplacian matrix of the power network, which is obtained when the voltage of the infinite node is constant and can thus be viewed as a ground node in the small-signal modeling. The specific form of matrix $\boldsymbol{Q}$ can be denoted by

$$
\boldsymbol{Q}=\left[Q_{i j}\right]_{(n+m) \times(n+m)}, Q_{i j}=\left\{\begin{array}{l}
-1 / \omega_{0} L_{i j}, i \neq j \\
\sum_{j=1}^{n+m+1} 1 / \omega_{0} L_{i j}, i=j
\end{array}\right.
$$

\section{APPENDIX B}

\section{TABLE B. I}

\section{PARAMETERS OF CONVERTERS}

Filter inductance, filter capacitance, dc capacitance: $0.05,0.05,0.038$
PI parameters of current control loop: 1,10
PI parameters of the constant active power control loop: $0.5,5$
PI parameters of the constant DC voltage control loop: $0.5,5$
Parameters of the voltage feedforward filter: 0.01
PI parameters of the PLL: 26,7800
Parameters of active power output and q-axis current reference: 1,0
Rated capacity of converters in per unit: 1

TABLE B.II

\begin{tabular}{|c|c|c|}
\hline \multicolumn{3}{|c|}{$\begin{array}{l}\text { Base capacity of ac system }(S / \mathrm{kVA}): 1500 \\
\text { Base voltage of ac system }\left(U_{B} / \mathrm{V}\right): 690 \\
\text { Reactance of unit length line in } 35 \mathrm{kV}(\Omega / \mathrm{km}): 0.38 \\
\text { Line kength between adjacent PLL-based converters }(\mathrm{km}): 0.28 \\
\text { Rated capacity of each converter (p.u.): } 1\end{array}$} \\
\hline \multicolumn{3}{|c|}{ The transformers parameters } \\
\hline & $220 / 35 \mathrm{kV}$ & $35 / 0.69 \mathrm{kV}$ \\
\hline Rated capacity (MVA) & 210 & 2 \\
\hline Percentage of short circuit voltage $(\%)$ & 14.28 & 6.5 \\
\hline
\end{tabular}

PARAMETERS IN THE 33-CONVERTER SYSTEM

\section{REFERENCES}

[1] L. Fan and Z. Miao, "An explanation of oscillations due to wind power plants weak grid interconnection," IEEE Trans. Sustain. Energy, vol. 9, no.1, pp. 488-490, Jan. 2018.

[2] L. Fan and Z. Miao, "Nyquist-stability-criterion-based SSR explanation for type-3 wind generators," IEEE Trans. Energy Conver., vol. 27, no. 3, pp. 807-809, Sept. 2012.

[3] A. Egea-Alvarez, S. Fekriasl, F. Hassan, and O. Gomis-Bellmunt, "Advanced vector control for voltage source converters connected to weak grids," IEEE Trans. Power Syst., vol. 30, no. 6, pp. 3072-3081, Nov. 2015.

[4] L. P. Kunjumuhammed, B. C. Pal, C. Oates, et al., "Electrical oscillations in wind farm systems: analysis and insight based on detailed modeling," IEEE Trans. Sustain. Energy, vol. 7, no. 1, pp. 51-62, Jan. 2016.

[5] M. Zhao, X. Yuan, J. Hu, and Y. Yan, "Voltage dynamics of current control time-scale in a VSC-connected weak grid," IEEE Trans. Power syst., vol. 31, no. 4, pp. 2925-2937, 2016.

[6] J. Z. Zhou, H. Ding, S. Fan, et al., "Impact of short-circuit ratio and phaselocked-loop parameters on the small-signal behavior of a VSC-HVDC converter," IEEE Trans. Power Del., vol. 29, no. 5, pp. 2287-2296, Oct. 2014.

[7] D. Dong, B. Wen, D. Boroyevich, P. Mattavelli and Y. Xue, "Analysis of phase-locked loop low-frequency stability in three-phase grid-connected power converters considering impedance interactions," IEEE Trans. Ind. Electron., vol. 62, no. 1, pp. 310-321, Jan. 2015.

[8] H. Liu, X. Xie, J. He, et al., "Subsynchronous interaction between directdrive PMSG based wind farms and weak AC networks," IEEE Trans. Power Syst., vol. 32, no. 6, pp. 4708-4720, Nov. 2017.

[9] Y. Li, L. Fan and Z. Miao, "Wind in weak grids: low-frequency oscillations, subsynchronous oscillations, and torsional interactions," IEEE Trans. Power Syst., vol. 35, no. 1, pp. 109-118, Jan. 2020.

[10] Du W, Dong W, Wang H, "A method of reduced-order modal computation for planning grid connection of a large-scale wind farm," IEEE Trans. Sustain. Energy, vol. 11, no. 3, pp. 1185-1198, July 2020.

[11] W. Du, X. Chen and H. Wang, "A method of open-loop modal analysis to examine the SSOs in a multi-machine power system with multiple variable-speed wind generators," IEEE Trans. Power Syst., vol. 33, no. 4, pp. 4297-4307, July 2018. 
[12] L. Harnefors, M. Bongiorno and S. Lundberg, "Input-admittance calculation and shaping for controlled voltage-source converters," IEEE Trans. Ind. Electron., vol. 54, no. 6, pp. 3323-3334, Dec. 2007.

[13] J. Sun, "Impedance-based stability criterion for grid-connected inverters," IEEE Trans. Power Electron., vol. 26, no. 11, pp. 3075-3078, Nov. 2011.

[14] L. Piyasinghe, Z. Miao, J. Khazaei and L. Fan, "Impedance model-based SSR analysis for TCSC compensated type-3 wind energy delivery systems," IEEE Trans. Sustain Energy, vol. 6, no. 1, pp. 179-187, Jan. 2015.

[15] W. Du, Y. Wang, H. Wang and X. Xiao, "Reduced-order method for detecting the risk and tracing the sources of small-signal oscillatory instability in a power system with a large number of wind farms," IEEE Trans. Power Syst., vol. 36, no. 2, pp. 1563-1582, March 2021.

[16] Y. Zhou, L. Zhao and W. Lee, "Robustness analysis of dynamic equivalent model of DFIG wind farm for stability study," IEEE Trans. Ind. Appl., vol. 54, no. 6, pp. 5682-5690, Nov. 2018.

[17] M. Ali, I. IIie, J. Milanovic, et al., "Wind farm model aggregation using probabilistic clustering," IEEE Trans. Power Syst., vol. 28, no. 1, pp. 309316, Feb. 2013.

[18] W Du, W. Dong and H F Wang, "Dynamic aggregation of same wind turbine generators in parallel connection for studying oscillatory stability of a wind farm," IEEE Trans. Power Syst., vol. 34, no. 6, pp. 4694-4705, Nov. 2019.

[19] M. El-Sharkawi, "Dynamic equivalent models for wind power plants," in Proc. IEEE Power Energy Soc. General Meeting, Detroit, MI, USA, Jul. 2011, pp. 1-5.

[20] I. Erlich, F. Shewarega, C. Feltes, F. Koch and J. Fortman, "Determination of dynamic wind farm equivalents using heuristic optimization," in Proc. IEEE PES General Meeting, San Diego, CA, USA, Jul. 2012, pp. 1-8.

[21] W. Dong, H. Xin, D. Wu and L. Huang, "Small signal stability analysis of multi-Infeed power electronic systems based on grid strength assessment," IEEE Trans. Power Syst., vol. 34, no. 2, pp. 1393-1403, March 2019.

[22] Huang L, Xin H, Li Z, et al., "Identification of generalized short-circuit ratio for on-line stability monitoring of wind farms," IEEE Trans. Power Syst., vol. 35, no. 4, pp. 3282-3285, July 2020.

[23] Dorfler F, Bullo F. "Kron reduction of graphs with applications to electrical networks," IEEE Transactions on Circuits \& Systems I Regular Papers, vol. 60, no. 1, pp. 150-163, Jan. 2013.

[24] G. W. Stewart, "Matrix perturbation theory," 1990.

[25] IEEE PES task force on Benchmark systems for stability controls, November 19, 2013. Available: http://www.sel.eesc.usp.br/ieee/ 